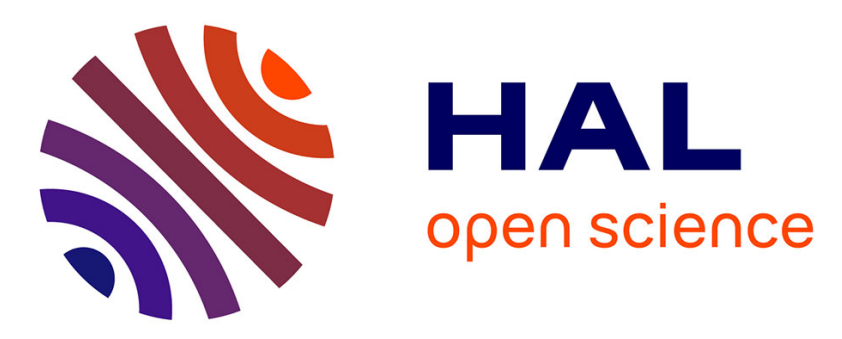

\title{
MIMO radar transmit array fed by a 1 x M passive chaotic cavity
}

Ettien Lazare Kpré, Thomas Fromenteze, Cyril Decroze

\section{To cite this version:}

Ettien Lazare Kpré, Thomas Fromenteze, Cyril Decroze. MIMO radar transmit array fed by a 1 x M passive chaotic cavity. The 10th European Conference on Antennas and Propagation, to be held in Davos, Switzerland, on 10-15 April 2016., Apr 2016, DAVOS, Switzerland. hal-01306477

\section{HAL Id: hal-01306477 \\ https://hal-unilim.archives-ouvertes.fr/hal-01306477}

Submitted on 24 Apr 2016

HAL is a multi-disciplinary open access archive for the deposit and dissemination of scientific research documents, whether they are published or not. The documents may come from teaching and research institutions in France or abroad, or from public or private research centers.
L'archive ouverte pluridisciplinaire HAL, est destinée au dépôt et à la diffusion de documents scientifiques de niveau recherche, publiés ou non, émanant des établissements d'enseignement et de recherche français ou étrangers, des laboratoires publics ou privés. 


\title{
MIMO radar transmit array fed by a $1 \times M$ passive chaotic cavity
}

\author{
Ettien L. Kpré ${ }^{1}$, Thomas Fromenteze ${ }^{1}$, Cyril Decroze ${ }^{1}$, \\ ${ }^{1}$ XLIM Research Institute - CNRS 123, Avenue Albert Thomas, 87060 Limoges Cedex, France \\ e-mail : ettien.kpre@etu.unilim.fr
}

\begin{abstract}
It is well known that MIMO radar waveforms should be orthogonal to probe simultaneously the MIMO channel matrix $H$. This method is not easily implementable since it requires as much transmitter chains as antennas. Herein, a compressive technique is introduced to measure the channel information avoiding multiple waveforms generation. This technique is thus based on the generation of a single waveform to probe the channel by the mean of a passive chaotic component connected to the transmit array. Mathematical formulations are presented considering a device with uncorrelated transfer functions allowing the transmission of orthogonal waveforms. Simulation and experimental results are presented to highlight the advantages of such a technique.
\end{abstract}

Index Terms-MIMO Radar, passive chaotic microwave component.

\section{INTRODUCTION}

MIMO radar became more popular and attracted much attention thanks to its ability to enhance radar performance [1]. The essence of this concept is the possibility to probe simultaneously the channel with $M$ orthogonal signals and record the backscattered signals with $N$ receiving antennas as depicted in Fig. 1. The received signal can thus be reassigned to the sources by performing correlation in pairs. The channel matrix $\mathbf{H}_{M \times N}$ represents the interaction with targets measured at each frequency, required for the image reconstruction [2]. Hence, each transmitting antenna should be addressed by a single waveform generated by a transmitter chain imposing the use of sophisticated and redundant RF systems.

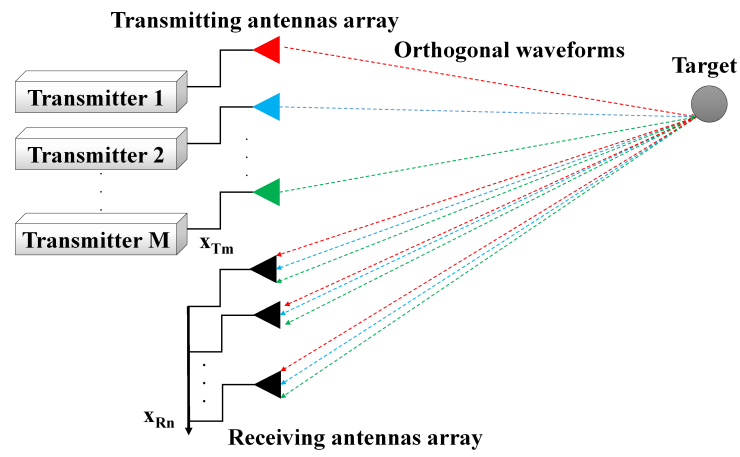

Fig. 1. Illustration of a conventional $M \times N$ MIMO radar. Each transmit antenna is fed by a transmitter. $x_{T m}$ and $x_{R n}$ are respectively the transmit and receive antenna positions.

In this article, a single input MIMO radar is proposed to reduce the number of transmitters maintaining the same number of antennas. The principle is based on the use of a chaotic microwave component in which transfer functions are uncorrelated. The first approach introduced in [3] was based a time reversal technique applied to a $1 \times 4$ small reverberation cell beamformer connected to UWB antennas. With a single input waveform, the authors demonstrated the control of antenna weightings without the need of active components or moving parts. This paper proposes the extension of this technique to MIMO radar transmit arrays. In this context, a single waveform is generated at the input of a chaotic component, and each transmit antenna can be addressed independently leading to the reduction of the number of transmitters compared to the conventional MIMO radar architectures. It is shown in this paper that the channel matrix can thus be retrieved from the chaotic component's transfer function matrix inversion. The remainder of this paper is organized as follows. Section II describes the conventional MIMO radar principle. Section III introduces the principle of unique input channel MIMO radar and the mathematical formulations ensuing. Simulation and experimental results are presented in section IV and V to present the advantages of the proposed method. Finally, section VI concludes this paper.

\section{Conventional MiMO RADAR}

Let us consider a radar architecture as depicted in Fig. 1. Ideally, the radiating aperture array consists of $M$ transmitting antennas radiating orthogonal waveforms and $N$ receiving antennas. However, in practice, the generation of orthogonal waveforms can hardly be achieved by real-life hardwares. Numerous papers have thus been focused on MIMO radar pseudo-orthogonal waveform design [4]-[5]. For example, the waveforms considered in this paper are frequency hopping modulated signals that can be computed considering Eq. (1):

$$
s(t)=\sum_{q=0}^{Q-1} u(t-q T c) \cdot \exp \left(j 2 \pi\left(f_{0}+c(q) \Delta f\right) t\right)
$$

where:

$$
u(t)= \begin{cases}1, & \text { if } 0<t<T c \\ 0, & \text { otherwise }\end{cases}
$$

and $c(q)$ is the $q^{\text {th }}$ element scrambled by a pseudo-noise Gold sequences [6], and $Q$, the number of frequencies. $T c$ corresponds to the pulse duration and is fixed by the total 
bandwidth $B w$ of the signal. The echo signals can be reassigned to the sources by performing correlation in pairs. The aim of the post processing is to estimate the channel matrix $[\mathbf{H}(f)]_{M \times N}$ which represents the interaction between the transmitting and receiving antennas:

$$
\mathbf{H}=\left(\begin{array}{cccl}
h_{11} & h_{12} & \ldots & h_{1 N} \\
h_{21} & h_{22} & \ldots & h_{2 N} \\
\ldots & \ldots & \ldots & \ldots \\
h_{M 1} & h_{M 2} & \ldots & h_{M N}
\end{array}\right)
$$

where the channel between the antennas $m$ and $n$ is :

$$
h_{m n} \propto \exp \left(j k\left(x_{T m}+x_{R n}\right) d \sin \theta_{0}\right)
$$

$x_{T m}$ and $x_{R n}$ are respectively the transmitting and the receiving antenna positions, $\theta_{0}$ refers to the signal Direction of Arrival (DoA) in azimutal plane considering the target at farfield distance from the radar system and $k$ the wavenumber. The received signal for each frequency sample $\mathbf{y}$ in the setup depicted in Fig. 1 is thus given by the equation bellow:

$$
\mathbf{y}=\mathbf{H}^{T} \mathbf{s}+\varepsilon
$$

where $\mathbf{S}$ and $\varepsilon$ are respectively the transmitted signal vector and the Gaussian additive noise. An estimation of channel matrix $\mathbf{H}_{\mathbf{r}}$ can be retrieved computing the pseudo-inverse $\mathbf{s}^{+}$ of the transmitted pseudo-orthogonal signals $\mathbf{s}$ :

$$
\begin{aligned}
\mathbf{H}_{r}^{T} & =\mathbf{y s}^{+} \\
& =\mathbf{H}^{T} \mathbf{s s}^{+}+\varepsilon \times \mathbf{s}^{+}
\end{aligned}
$$

Considering the pseudo-orthogonality of the radiated waveforms, the following relation holds :

$$
\mathbf{s s}^{+} \approx[I]_{M \times M}
$$

leading to a perfect estimation of $\mathbf{H}$ in a noiseless scenario. MIMO imaging algorithms [1]-[7] can then be applied to reconstruct the target image using the channel matrix $\mathbf{H}$. Since the transmitted waveforms should be orthogonal for a simultaneous measurement of the channel matrix, each antenna should be addressed by an independent single waveform. As consequence, the number of transmitters increases with the number of transmit antennas needed.

In this context, the main contribution of this paper is introduction of a comparable technique achieved in the physical layer, allowing for a simplification of the MIMO RF architectures using a single transmitter to feed the $M$ transmit antennas. The following section introduces this principle.

\section{SINGLE INPUT MIMO RADAR PRINCIPLE}

The technique presented here is based on a single input transmit array. The orthogonality of the transmitted waveforms is reached by the propagation in a $1 \times M$ microwave cavity connected to the antennas. A generic setup is described in Fig. 2.

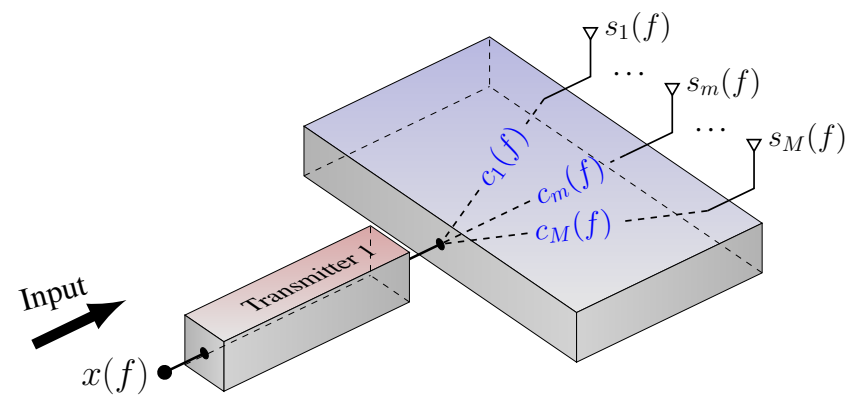

Fig. 2. Illustration of MIMO Radar transmitters feeding with $1 \times M$ passive microwave cavity. Only one RF chain is needed in opposition with the conventional MIMO transmitter array.

The transmitted signals $s(t)$ must be pseudo-orthogonal to estimate the channel matrix $\mathbf{H}$. This requirement imposes a constraint on the component transfer functions which should be uncorrelated. This feature is the key factor of the single port MIMO radar. This is reachable with a metallic microwave oversized cavity which presents a high frequency diversity [3]. In the depicted Fig. 2, the signal $s_{m}(f)$ radiated by the antenna $m$ is :

$$
s_{m}(f)=x(f) c_{m}(f)
$$

where $x(f)$ is a signal with good auto-correlation properties generated at the input of the component, and $c_{m}(f)$ the component $m^{\text {th }}$ transfer function. For the sake of notation simplicity, Eq. (8) can be presented in a matrix form:

$$
\mathbf{s}=x \mathbf{c}
$$

Therefore, the signals measured by the receiving antenna array considering a noise less scenario is:

$$
\mathbf{y}=\mathbf{H}^{T} \mathbf{s}
$$

with $\mathbf{H}$ the previously defined channel matrix which accounts for useful informations required for the microwave imaging. An estimation of the channel matrix can be computed at each frequency by compensating the contribution of the generated waveform $x$ and the component transfer function $\mathbf{c}$ in a single operation:

$$
\mathbf{H}_{r}^{T}=\mathbf{y s}^{+}
$$

where $\mathbf{s}^{+}$is the transmitted waveform pseudo-inverse. Numerous regularization techniques can be applied to solve this linear problem, such as Tikhonov regularization giving the following pseudo-inverse expression:

$$
\mathbf{s}_{\mu}^{+}=\left(\mathbf{s}^{\dagger} \mathbf{s}+\mu I\right)^{-1} \mathbf{s}^{\dagger}
$$

where $\mu$ denotes the regularization parameter which is used as a threshold to prevent any ill-conditioning problem [8]. (. $)^{\dagger}$ denotes the transposed-conjugate operator. The expression of the estimated channel matrix can be expressed by: 


$$
\begin{aligned}
\mathbf{H}_{r \mu}^{T} & =\mathbf{y} \mathbf{s}_{\mu}^{+} \\
& =\mathbf{H}^{T} \mathbf{s s}_{\mu}^{+} \\
& \approx \mathbf{H}^{T} \mathbf{R}_{\mu}
\end{aligned}
$$

Considering an ideal equalization of the generated signal $\mathbf{x}$, $\mathbf{R}_{\mu}$ can be considered as the correlation matrix of the transfer functions:

$$
\mathbf{R}_{\mu}=\left[\begin{array}{ccll}
c_{1} \times c_{1 \mu}^{+} & c_{1} \times c_{2 \mu}^{+} & \ldots & c_{1} \times c_{m \mu}^{+} \\
c_{2} \times c_{1 \mu}^{+} & c_{2} \times c_{2 \mu}^{+} & \ldots & c_{2} \times c_{m \mu}^{+} \\
\ldots & \ldots & \ldots & \ldots \\
c_{m} \times c_{1 \mu}^{+} & c_{m} \times c_{2 \mu}^{+} & \ldots & c_{m} \times c_{m \mu}^{+}
\end{array}\right]
$$

Ideally, this matrix tends to an identity matrix, corresponding to a perfectly orthogonal transfer functions leading to a perfect estimation of the channel matrix $\mathbf{H}_{r \mu}^{T}=\mathbf{H}^{T}$. Such constraints are not practically achievable, limited by the frequency diversity of the chaotic component, but can be approached using a microwave cavity with regards to the operating wavelengths [9].

Therefore, only a single wideband signal is generated at the input of a chaotic microwave cavity. The pseudo-orthogonality of radiated waveforms is ensured by the pre-characterized device transfer functions. The next section presents a numerical comparison between a conventional MIMO array and the proposed method.

\section{NUMERICAL RESULTS}

A simulation of a radar experiment is presented to study the proposed method. The radar scenario consists of $M=4$ transmit and $N=4$ receiving antennas with inter-elements spacing respectively of $d T=1.4 \lambda_{c}$ and $d R=0.7 \lambda_{c}$. Where $\lambda_{c}$ is the central wavelength in the $2.5 \mathrm{Ghz}$ to $3.5 \mathrm{Ghz}$ band. The inter element spacing are chosen in order to exploit MIMO radar virtual array [7].

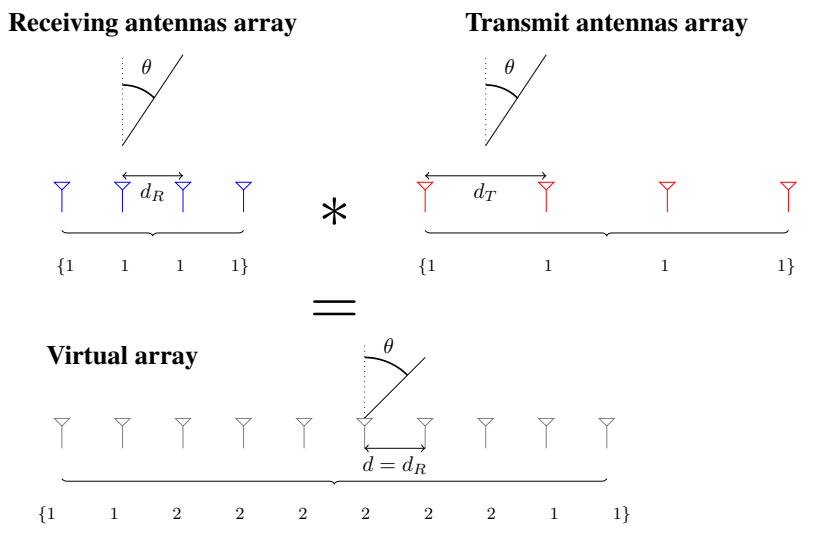

Fig. 3. MIMO Radar array. The virtual array is the convolution of the real transmit and receiving antennas aperture. The undermentioned numbers represent virtual elements redundancy.

A point-like target with an isotropic Radar Cross Section (RCS) is placed in the radar field of view (FOV) at $2 m$ in range and $0.5 \mathrm{~m}$ in cross-range which corresponds to an angle offset of $14^{\circ}$ in azimuth. The first simulation setup is a conventional MIMO radar in which the transmitted waveforms are modulated by $Q=32$ carriers and are computed via Eq.1. In the second scenario, the chaotic cavity impulse responses are simulated using Rayleigh distributed magnitude modulated by an exponential decay with a time decay $\tau=0.4 \mu \mathrm{s}$ which account for the quality factor of the cavity [10]. This simple mathematical model is used to reproduce the general behavior of an oversized cavity, leading to the following transfer function addressing the $m^{\text {th }}$ antenna :

$$
c_{m}(f)=\mathscr{F}\left[r_{m}(t) \exp (-t / \tau)\right]
$$

where $r_{m}(t)$ denotes a random Gaussian noise sequence and $\mathscr{F}$ is the Fourier transform. Fig. 4 shows the comparison between the conventional MIMO radar and the proposed MIMO technique.

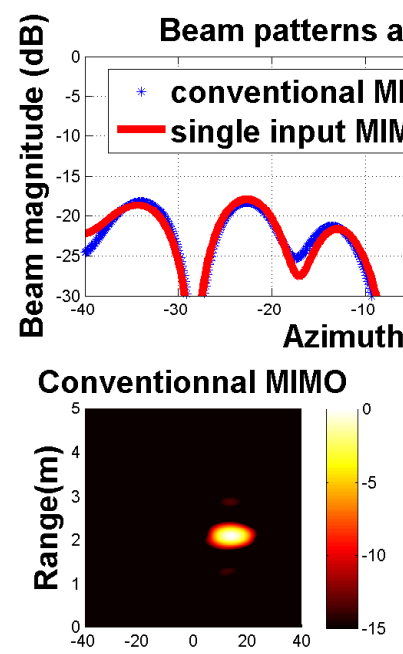

Azimuth angle (Degree) at the target range $R=2 m$

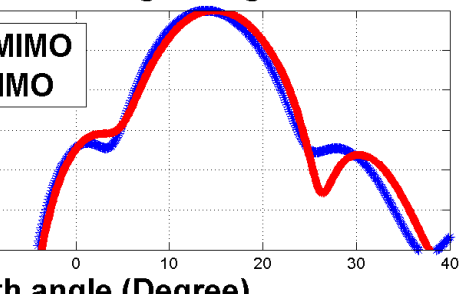

$1 \times 4$ compressed MIMO

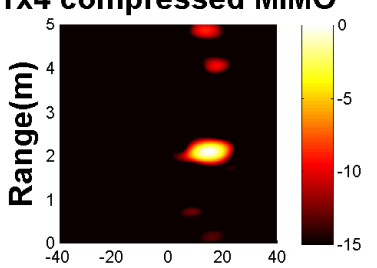

Azimuth angle (Degree)
Fig. 4. Comparison of the time domain beamforming in the conventional MIMO case and the proposed one.

Using the same bandwidth and the same post-processing technique. The results show that the proposed method can be an efficient alternative for the conventional MIMO architecture simplification. Despites a SNR reduction ( $4 d B$ compared to the conventional MIMO radar), the compressed MIMO method can reach the same performances of the conventional with the main advantages of saving orthogonal waveforms generation and architecture simplification. The resolution of the beam at $-3 d B$ of the maximum is the same for both techniques $\left(8.5^{\circ}\right)$. In this simulation conditions the dynamic (main lobe to the higher sidelobe ration) is almost the same in azimuth and is about $16 d B$. This setting depends mainly of the correlation levels of the component transfer functions.

\section{Measurement Results and discuss}

A measurement setup is set to match the simulation. An empty metallic microwave cavity with outer dimensions of $0.8 \times 0.8 \times 1 \mathrm{~m}^{3}$ is used to connect $M=4$ transmitters. The $N=$ 4 receivers are directly connected to an oscilloscope Agilent 
DSA90404A $20 \mathrm{GSa} / \mathrm{s}$. The inter elements spacing is $d T=$ $1.4 \times \lambda_{c}$ for the transmitting and $d R=0.7 \times \lambda_{c}$ for the receiving antennas. Fig. 5 shows the measurement setup.

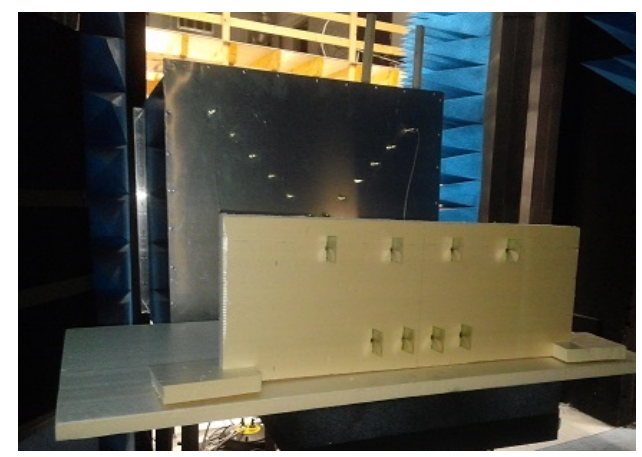

(a)

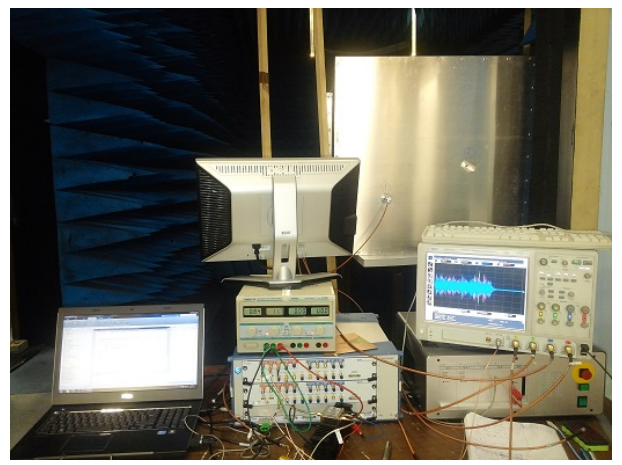

(b)

Fig. 5. Single input MIMO radar setup. (a) UWB Tapered Vivaldi antennas array. The transmitters are connected to the metallic cavity behind and the receivers are connected to an oscilloscope. (b) Measuring and post processing bench.

The metallic cavity impulse responses have been precharacterized as shown in Fig. 6. Good correlation properties are reached thanks to the frequency diversity features of the cavity.

An Arbitrary waveform generator Agilent M8190A 12 $\mathrm{GSa} / \mathrm{s}$ is used to generate the single waveform (from Eq. 1) at the input of the cavity, and the oscilloscope is used to directly measure the echo signals. A watter bottle is set in front of the radar as shown in Fig. 7.

The backscattered signals are measured and post-processed to retrieve the bottle position. Fig. 6.b-c show the beamforming results which uphold the feasibility of the proposed method. With a single generated waveform, the MIMO channel matrix can be measured in one shot. The beam width at $-3 d B$ of the maximum is about $8^{\circ}$ which tends to the theoretical one. The dynamic is $12 d B$ in the azimuth and the SNR of the image is about $18 d B$. This SNR is computed considering the real image and the background noise ratio.

\section{CONCLUSION}

In this paper, a technique implemented for the simplification of MIMO radar architectures has been proposed. Using a

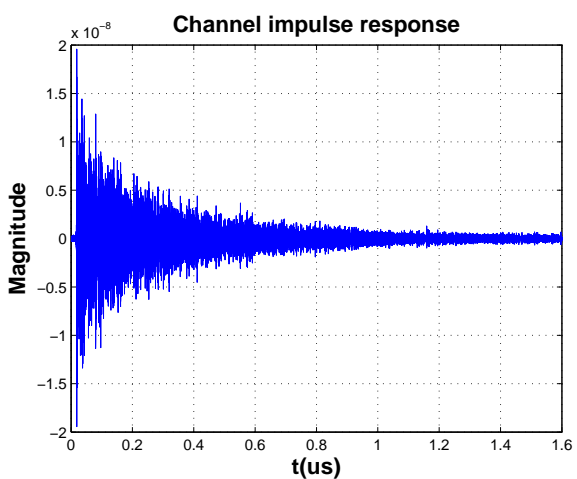

(a)

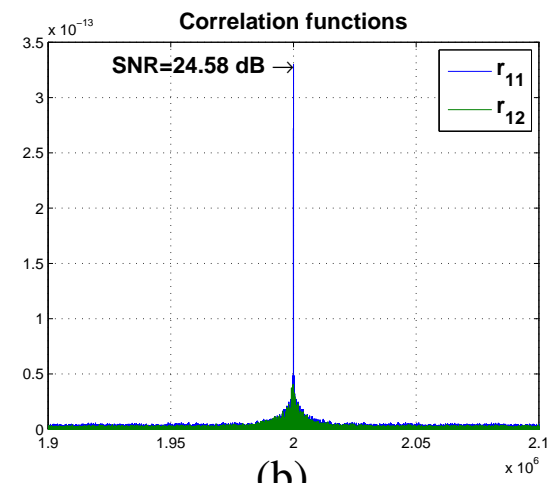

(b)

Fig. 6. Metallic cavity general behavior. (a) $1^{r s t}$ channel impulse response (b) $1^{r s t}$ channel autocorrelation $r_{11}$ and cross-correlation $r_{12}$ function between channel 1 and 2.

passive microwave component whose transfer functions are quasi-orthogonal, MIMO radar transmit antennas can be independently addressed via a single generated waveform. This condition has been achieved with an oversized microwave cavity in regards to the operating wavelengths. The simulation results show that the proposed method can be applied to save orthogonal waveforms generation and to minimize the complexity of the conventional MIMO architecture while maintaining the same number of antennas. Future works will focus on the design of miniaturized microwave components that can provide good correlation properties. Herein the component is applied only at the transmit array, but it can also be used at the receiving array such that only a unique signal is measured at the reception leading to a $2 \times M N$ ports passive component feeding $M \times N$ MIMO transmit/receiving antennas.

\section{REFERENCES}

[1] D. W. Bliss and K. W. Forsythe, "Multiple-input multiple-output (MIMO) radar and imaging: degrees of freedom and resolution", in Signals, Syst

[2] Li Jian and Stoica Petre, "MIMO radar signal processing". J. Wiley \& Sons 2009.

[3] D. Carsenat and C. Decroze, "UWB Antennas Beamforming Using Passive Time-Reversal Device," IEEE Antennas and Wireless Propagation Letters, vol. 11, pp. 779-782, 2012.

[4] W.-Q. Wang, "MIMO SAR OFDM Chirp Waveform Diversity Design With Random Matrix Modulation," IEEE Transactions on Geoscience and Remote Sensing, vol. 53, no. 3, pp. 1615-1625, Mar. 2015.

[5] G. Babur, O. A. Krasnov, A. Yarovoy, and P. Aubry, "Nearly Orthogonal Waveforms for MIMO FMCW Radar,". Aerospace and Electronic Systems, IEEE Transactions on, vol. 49, no. 3, pp. 1426-1437, 2013. 


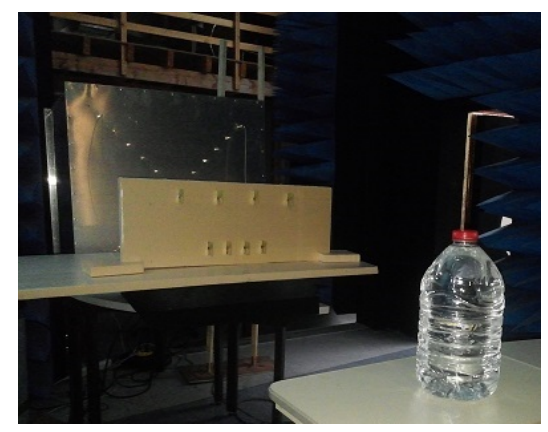

(a)

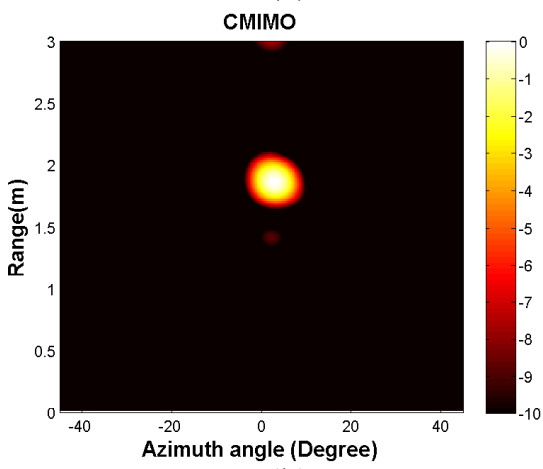

(b)

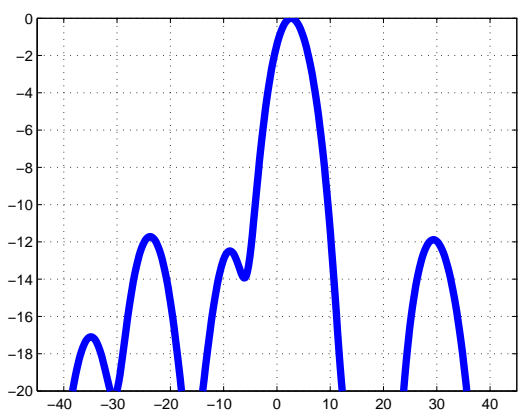

(c)

Fig. 7. Proposed MIMO radar measurement imaging results. (a) Radar scenario, a water bottle placed in front the Radar.(b) MIMO beamforming image. (c) Beam pattern at the target range $(R=1.8)$ plane.

[6] R. M. Buehrer. Code Division Multiple Access (CDMA). Morgan \& Claypool Publishers, 2006.

[7] E. L. Kpré, T. Fromenteze, C. Decroze, and D. Carsenat, "Experimental implementation of an ultra-wide band MIMO radar," in Radar Conference (EuRAD), 2015 European, 2015, pp. 89-92.

[8] T. Fromenteze, C. Decroze, and D. Carsenat, "Waveform Coding for Passive Multiplexing: Application to Microwave Imaging," IEEE Transactions on Antennas and Propagation, vol. 63, no. 2, pp. 593-600, Feb. 2015.

[9] Kildal, P.-S.; Rosengren, K., "Correlation and capacity of MIMO systems and mutual coupling, radiation efficiency, and diversity gain of their antennas: simulations and measurements in a reverberation chamber," in Communications Magazine, IEEE , vol.42, no.12, pp.104-112, Dec. 2004

[10] David A. Hill Electromagnetic Theory of Reverberation Chambers, Radio-Frequency Technology Division Electronics and Electrical Engineering Laboratory National Institute of Standards and Technology 325 Broadway Boulder, Colorado 80303-3328 